\title{
Hypermethylation of the IGF2 differentially methylated region 2 is a specific event in insulinomas leading to loss-of-imprinting and overexpression
}

\author{
Emelyne Dejeux ${ }^{1}$, Robert Olaso ${ }^{2}$, Bertrand Dousset ${ }^{3}$, Anne Audebourg ${ }^{4,5}$, \\ Ivo G Gut ${ }^{6}$, Benoit Terris ${ }^{4,5}$ and Jörg Tost $^{1}$
}

\footnotetext{
${ }^{1}$ Laboratory for Epigenetics CEA-Institut de Génomique, Centre National de Génotypage, 91000 Evry, France

${ }^{2}$ Gene Expression Team, Department of Translational Research, CEA-Institut de Génomique, Centre National de Génotypage, 91000 Evry, France

${ }^{3}$ Service de chirurgie digestive et endocrinienne and ${ }^{4}$ Service d'Anatomie et de Cytologie Pathologique, Hôpital Cochin, AP-HP, Université Paris Descartes, 75014 Paris, France

${ }^{5}$ Institut Cochin de Génétique Moléculaire, Université Paris V René Descartes, CNRS (UMR 8104), 75014 Paris, France

${ }^{6}$ Department of Translational Research, CEA-Institut de Génomique, Centre National de Génotypage, 91000 Evry, France

${ }^{7}$ Institut National de la Santé et de la Recherche Médicale U567, 75014 Paris, France
}

(Correspondence should be addressed to J Tost, Laboratory for Epigenetics, CEA-Institut de Génomique, Centre National de Génotypage, Bâtiment G2, 2 rue Gaston Crémieux, CP 5721, 91057 Evry Cedex, France; Email: tost@cng.fr)

\begin{abstract}
Prediction of the evolution of endocrine pancreatic tumors remains difficult based on histological criteria alone. We have previously demonstrated that epigenetic changes are an early event in a mouse model developing insulinomas. Particularly, overexpression of the imprinted IGF2 was caused by the hypermethylation of $\mathrm{CpGs}$ in the differentially methylated region 2 (DMR2). Here, we investigated whether IGF2 hypermethylation is also observed in human insulinomas and whether this alteration is common to other human endocrine tumors of the pancreas and the digestive tract. We analyzed the methylation status of $40 \mathrm{CpGs}$ located in the DMR0 and DMR2 of the IGF2 as well as in the H19DMR by pyrosequencing in a cohort of 62 patients with pancreatic or small intestine endocrine tumors. Altered methylation patterns were observed in all tumor types for the different regions of IGF2, but not for H19. However, hypermethylation of the IGF2 DMR2 was specific for insulinomas and did not occur in any of the other types of tumors which were characterized by a loss of methylation in this region. Gain of methylation in the IGF2 DMR2 in insulinomas correlated with loss-of-imprinting and promoter 4 mediated overexpression of IGF2 at the RNA and protein level. Furthermore, a decreasing degree of methylation in the different regions of IGF2 correlated well with increasing degree of malignancy according to the WHO classification of pancreatic endocrine tumors (PETs), suggesting that methylation of IGF2 might be a useful biomarker for classification and staging of PETs.
\end{abstract}

Endocrine-Related Cancer (2009) 16 939-952

\section{Introduction}

Pancreatic endocrine tumors (PETs) represent 1-2\% of pancreatic neoplasms, but are of great clinical interest due to their heterogeneous evolutionary potential (Halfdanarson et al. 2008). They are classified into functioning or non-functioning tumors based on their clinical manifestations. Functioning tumors are associated with clinical syndromes caused by inappropriate secretion of hormones such as gastrinomas and insulinomas, the latter being the most frequent islet cell tumor of the pancreas (Grant 2005, Halfdanarson et al. 2008). PETs occur sporadically or in association with genetic syndromes such as the multiple endocrine neoplasia type 1 (MEN1) syndrome caused by mutations in MEN1 and characterized by multifocal endocrine tumors affecting the endocrine pancreas, parathyroids, anterior 
pituitary, adrenal, and thyroid glands (Zikusoka et al. 2005). Different genetic and chromosomal alterations have been implicated in the pathogenesis of endocrine tumors (reviewed in Zikusoka et al. (2005)), and it has recently been shown that epigenetic alterations might play an equally important part during tumorigenesis. DNA methylation plays a key role in the regulation of gene expression, and altered DNA methylation patterns have been observed in several diseases, particularly cancer where genome-wide hypomethylation coincides with gene-specific hypermethylation (Jones \& Baylin 2007). Hypermethylation of the promoter region of tumor-suppressor genes including CDKN2A (p14, p16), COX2, RASSF1A, hMLH1, $M G M T, E S R 1$, and $R A R-\beta$ has been observed in several studies investigating pancreatic and ileal endocrine tumors (Serrano et al. 2000, Chan et al. 2003, House et al. 2003b, Liu et al. 2005, Choi et al. 2007). Methylation changes including gene-specific hypermethylation and global hypomethylation are generally more pronounced in tumors of the digestive tract than in PETs (Chan et al. 2003, Liu et al. 2005, Choi et al. 2007). Presence of methylation at gene promoters in general and at the $C D K N 2 A$ and RASSF1A promoters, in particular, has been associated with tumor recurrence, more advanced tumor stage, presence of metastases, and shorted survival (House et al. 2003b, Liu et al. 2005), while hMLH1 methylation associated with microsatellite instability and prolonged survival (House et al. 2003a).

Imprinted genes are a subgroup of genes that might be particularly sensitive to DNA methylation alterations during tumorigenesis. Their parent-of-origindependent mono-allelic expression pattern is at least partially controlled by CpG-rich differentially methylated regions (DMRs; Reik et al. 2001). The insulin-like growth factor 2 (IGF2)-H19 locus at chromosome $11 \mathrm{p} 15.5$ is a paradigm of human imprinting (Lewis \& Reik 2006). The $I G F 2$ is expressed from the paternally inherited allele only and plays a key role in mammalian growth and apoptosis influencing fetal cell division and differentiation (Jones \& Clemmons 1995). The H19 gene, located $135 \mathrm{~kb}$ towards the telomere, is oppositely imprinted and is expressed only from the maternal allele. The human IGF2-H19 locus is controlled by three DMRs, the IGF2 DMR0 which is located between exons 2 and 3, the DMR2 (between exons 8 and 9), and the $H 19$ DMR located $4 \mathrm{~kb}$ upstream of the transcription start of H19, which is the imprinting control region (ICR) of the $I G F 2-H 19$ locus (Fig. 1A). The H19 ICR contains seven binding sites for the zinc finger protein CTCF, which acts as methylation-sensitive insulator. On the unmethylated maternal allele, the insulator protein binds to the ICR and forms - at least in mice - higher order chromatin loops that block the access of the enhancers to the Igf 2 promoters (Murrell et al. 2004). On the paternal allele, however, DNA methylation blocks CTCF binding and permits the enhancers to access the $I G F 2$ promoters resulting in $I G F 2$ expression. Alterations of the methylation patterns of the H19 ICR lead to lossof-imprinting (LOI) and disruption of the allelespecific gene expression patterns of the entire locus. These changes have been observed in growth disorders such as Beckwith-Wiedemann and Silver-Russell syndrome (Gicquel et al. 2005, Eggermann et al. 2008) and in Wilms tumor, where biallelic IGF2 expression is accompanied by de novo methylation and silencing of the maternal $\mathrm{H} 19$ allele (Bjornsson et al. 2007). However, LOI of $I G F 2$ leading to pathological biallelic expression but without alterations of the $\mathrm{H} 19$ ICR is a common phenomenon in cancer, and has been observed in many human cancers including breast and colorectal cancers (Cui et al. 2003, Kaneda \& Feinberg 2005, Ito et al. 2008). Although changes in IGF2 methylation and expression have to our knowledge not yet been studied in human PETs, several studies hint at a possible implication of IGF2 in the pathogenesis of pancreatic endocrine neoplasms and more specifically insulinomas.

Igf 2 was biallelically expressed in pancreatic $\beta$-cells of a mouse model in which the SV40 large T-antigen was under control of the rat insulin regulatory region, alleles were activated early during tumorigenesis and the level of expression was found to correlate with hyperproliferation and tumor growth (Christofori et al. 1994, 1995). We have previously shown that mice with a conditional knockout of Menl in pancreatic $\beta$-cells develop insulinomas (Fontaniere et al. 2006). Six CpGs in the core region of the Igf2 DMR2 were significantly hypermethylated in these mice leading to overexpression of the gene probably through increased levels of transcription initiation (Murrell et al. 2001, Fontaniere et al. 2006).

Here, we investigated whether the methylation and expression changes observed in the different mouse models are also present in human insulinomas and/or other human endocrine tumors of the pancreas or the digestive tract. We found hypermethylation of the IGF2 DMR2 to be a specific event in human insulinomas, which does not occur in any of the other types of endocrine tumors that are characterized by a loss of methylation at this locus. The increase in methylation in insulinomas leads to a LOI of IGF2 and correlates with $I G F 2$ overexpression at the RNA and protein level. Most interestingly, the methylation level 


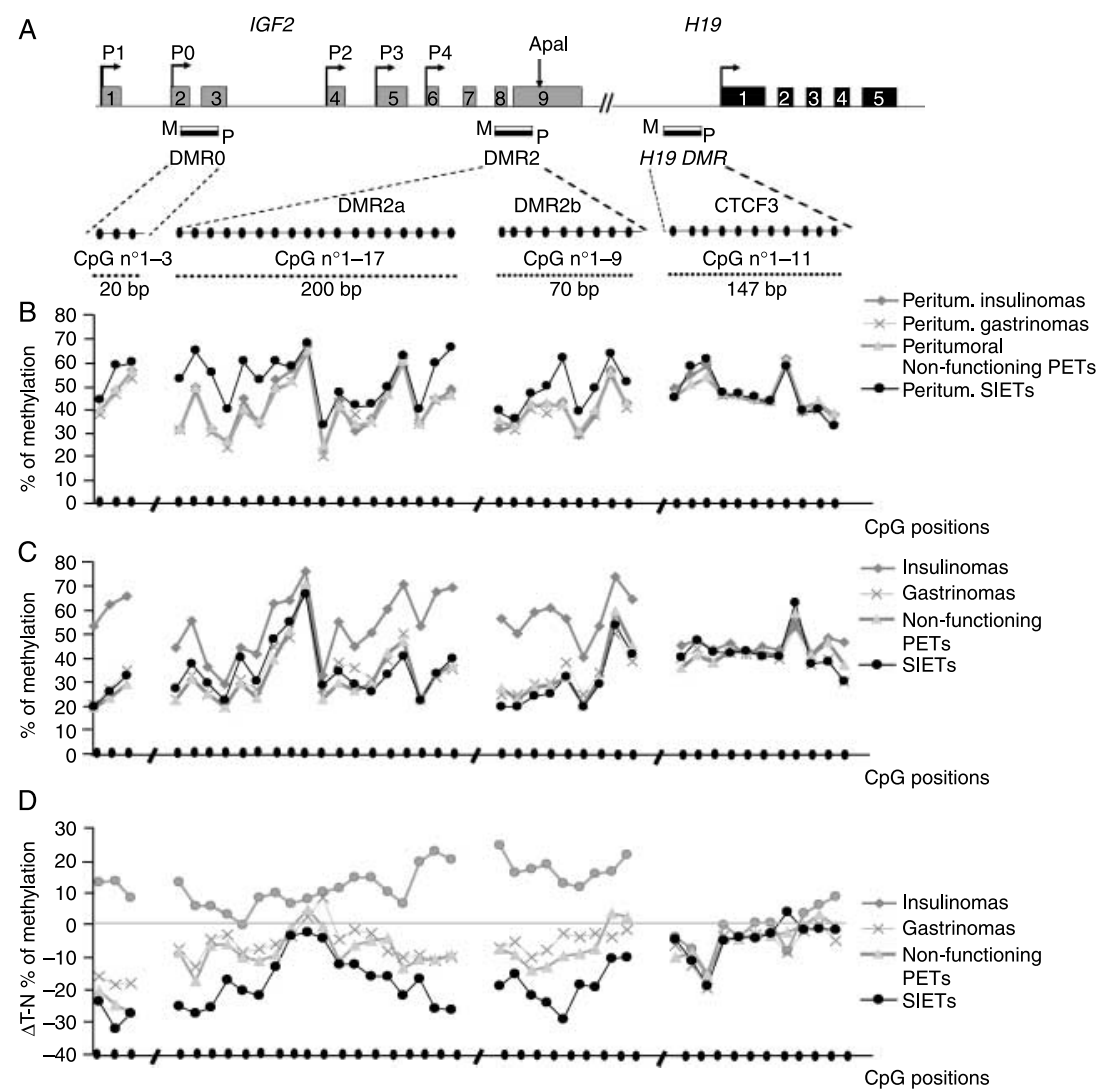

Figure 1 (A) Structural characteristics of the human IGF2-H19 locus with nine exons and five exons for IGF2 and H19 respectively (grey numbered squares). Transcription start and promoters used are indicated by arrows. Regions of differential methylation (DMRs) are shown with black (methylated allele) and white (unmethylated allele) bars. IGF2 DMR0 (3 CpGs) and IGF2 DMR2 (DMR2a $=17 \mathrm{CpG}$ and DMR2b=9 CpGs) were analyzed. For the $\mathrm{H} 19 \mathrm{DMR}$, the third CTCF-binding site containing $11 \mathrm{CpGs}$ is represented. For each DMR, the number of $\mathrm{CpG}$ s analyzed is indicated and their relative positions are represented by filled circles. The number of $b p$ analyzed is indicated under each region. ( $p$, paternal; $m$, maternal). Overview of the methylation patterns of the IGF2-H19 locus (expressed in percentage of the mean methylation values for each $\mathrm{CpG}$ ) in normal adjacent tissue (B) and their corresponding tumors (C). (D) The differential DNA methylation for each tumor type between the average tumoral DNA methylation patterns and the peritumoral methylation profile is shown. Details on the average DNA methylation degree for each region as well as the S.D. and range of methylation values are given in the Supplementary Table 2, which can be viewed online at http://erc.endocrinology-journals.org/supplemental/.

of the IGF2 DMRs correlated well with the WHO classification for PETs with methylation levels decreasing with increasing grade of malignancy.

\section{Materials and methods}

\section{Study group}

A total of 62 patients with endocrine tumors of the pancreas or the digestive tract were analyzed to investigate changes in the DNA methylation patterns. All patients of this study were submitted to surgical resections in the Hospital Cochin, Paris, France. Tumor samples were collected in accordance with French law and ethical guidelines and their use approved by the institutional ethics committee. Analyzed samples included 11 insulinomas and 5 normal adjacent pancreatic tissues, 12 gastrinomas and 7 normal adjacent pancreatic tissues and 19 non-functioning PETs with 7 normal adjacent pancreatic tissues, and 18 small intestine endocrine tumors (SIETs) with 11 normal adjacent ileal mucosa (Table 1). The gastrinomas were either of pancreatic (seven tumors) or duodenal wall (five tumors) origin. PETs were classified and graded as benign, uncertain behavior, and welldifferentiated endocrine carcinomas according to the WHO criteria (Klöppel et al. 2004). Two of the nonfunctioning PETs and two gastrinomas had a MEN1 phenotype and MEN1 mutations were confirmed by mutation analysis.

For expression analysis, 19 corresponding RNA samples were available for 6 of the insulinomas, 4 of the gastrinomas, 3 of the non-functioning PETs, and 
Table 1 The main clinical characteristics of patient cohort

\begin{tabular}{|c|c|c|c|c|c|c|}
\hline & & Insulinomas & Gastrinomas & $\begin{array}{l}\text { Non-functioning } \\
\text { PETs }\end{array}$ & SIETs & Total \\
\hline $\begin{array}{c}\text { Number of } \\
\text { patients }\end{array}$ & & 12 & 12 & 20 & 18 & 62 \\
\hline \multirow[t]{3}{*}{ Age } & Min & 20 & 22 & 18 & 44 & 18 \\
\hline & Max & 76 & 68 & 75 & 80 & 80 \\
\hline & Average & 48.25 & 48.33 & 50 & 62.28 & 52.21 \\
\hline \multirow[t]{2}{*}{ Sex } & Male & 5 & 10 & 11 & 12 & 38 \\
\hline & Female & 7 & 2 & 9 & 6 & 24 \\
\hline Tumors & & 11 & 12 & 19 & 18 & 60 \\
\hline $\begin{array}{l}\text { Peritumoral } \\
\text { samples }\end{array}$ & & 5 & 7 & 7 & 11 & 30 \\
\hline $\begin{array}{l}\text { Number of } \\
\text { patients with } \\
\text { matched } \\
\text { tumor and } \\
\text { peritumoral } \\
\text { samples } \\
\text { available }\end{array}$ & & 4 & 7 & 6 & 11 & 28 \\
\hline \multirow[t]{2}{*}{ Metastases } & Positive & 0 & 10 & 14 & 18 & 42 \\
\hline & Negative & 11 & 2 & 5 & 0 & 18 \\
\hline \multirow{3}{*}{$\begin{array}{l}\text { World Health } \\
\text { Organization } \\
\text { classification }\end{array}$} & Benign & 5 & 0 & 0 & - & 5 \\
\hline & Uncertain behavior & 6 & 2 & 5 & - & 13 \\
\hline & $\begin{array}{l}\text { Well-differentiated } \\
\text { endocrine carcinoma }\end{array}$ & 0 & 10 & 14 & - & 24 \\
\hline
\end{tabular}

5 SIETs. A pancreatic RNA sample from an individual without signs of tumor development was obtained to determine the expression level of IGF2 in normal pancreas, and expression levels were further verified using two commercial pancreatic RNA preparations from two different lots originating from Caucasian males aged 35 (Clontech).

\section{Preparation of DNA for methylation analysis}

DNAs were isolated from frozen tissue samples using standard phenol-chloroform extraction. DNA concentrations were determined using the Quant-iT dsDNA broad range or high sensitivity assay kit (Molecular Probes, Invitrogen) on a SpectraMAX Gemini XPS microplate spectrofluorometer (Molecular Devices, St Grégoire, France) and normalized to a concentration of $50 \mathrm{ng} / \mu \mathrm{l}$. Genomic DNA of $1 \mu \mathrm{g}$ was treated with sodium bisulphite using the EpiTect 96 Bisulfite Kit (Qiagen) according to the manufacturer's instructions.

\section{Methylation assays by pyrosequencing}

Quantitative DNA methylation analysis of the bisulphite-treated DNA was performed by pyrosequencing or - in case of several sequencing primers - by serial pyrosequencing (Tost \& Gut 2007). Particular attention was paid to the choice of primer sets since a large number of single nucleotide polymorphisms (SNP) was described in these regions. Regions of interest were amplified using $25 \mathrm{ng}$ of bisulfite-treated human genomic DNA and 5 pmol of forward and reverse primer, one of them being biotinylated. Oligonucleotides for PCR amplification and pyrosequencing (see Supplementary Table 1, which can be viewed online at http://erc.endocrinology-journals.org/ supplemental/) were synthesized by Biotez (Buch, Germany). Analysis of repetitive elements as a surrogate measure of global DNA methylation changes was performed as previously described (Yang et al. 2004). Standard reaction conditions were $1 \times$ HotStar Taq buffer supplemented with $1.6 \mathrm{mM} \mathrm{MgCl}_{2}, 100 \mu \mathrm{M}$ dNTPs, and 2.0 U HotStar Taq polymerase (Qiagen) in a $25 \mu \mathrm{l}$ volume. The PCR program consisted of a denaturing step of $15 \mathrm{~min}$ at $95^{\circ} \mathrm{C}$ followed by 50 cycles of $30 \mathrm{~s}$ at $95{ }^{\circ} \mathrm{C}, 30 \mathrm{~s}$ at the respective annealing temperature (see the Electronic Supplementary Table 1), and $20 \mathrm{~s}$ at $72{ }^{\circ} \mathrm{C}$, with a final extension of $5 \mathrm{~min}$ at $72{ }^{\circ} \mathrm{C}$. Some assays had particular PCR conditions that are detailed in the Electronic Supplementary Table 1 . After verification by standard gel electrophoresis on a $2 \%$ agarose gel, $5 \mu \mathrm{l}$ of PCR product were incubated, for $10 \mathrm{~min}$ at room 
temperature with shaking, in the presence of $40 \mu \mathrm{l}$ of binding buffer $(10 \mathrm{mM}$ Tris, $2 \mathrm{M} \mathrm{NaCl}, 1 \mathrm{mM}$ EDTA, 0.1\% Tween 20; pH 7.6; adjusted with $1 \mathrm{M}$ $\mathrm{HCl}), 2 \mu \mathrm{l}$ of streptavidin-coated sepharose beads (GE Healthcare, Uppsala, Sweden), and $33 \mu \mathrm{l}$ of $\mathrm{ddH}_{2} \mathrm{O}$. The binding mix was purified and rendered single stranded using the Pyrosequencing Vacuum Prep Workstation (Pyrosequencing AB, Uppsala, Sweden) according to the manufacturer's instructions. Beads were released into $12 \mu \mathrm{l}$ annealing buffer (20 mM Tris, $2 \mathrm{mM} \mathrm{Mg-acetate;} \mathrm{pH} \mathrm{7.6;} \mathrm{adjusted}$ with $4 \mathrm{M}$ acetic acid) containing 4 pmol of the respective sequencing primer (see the Electronic Supplementary Table 1). Primers were annealed to the target by incubation at $80{ }^{\circ} \mathrm{C}$ for $2 \mathrm{~min}$. Quantitative DNA methylation analysis was carried out on a PSQ 96MD system with the PyroGold SQA Reagent Kit (Pyrosequencing), and results were analyzed using the Q-CpG software (V.1.0.9, Pyrosequencing).

\section{mRNA expression analysis}

RNAs were isolated from frozen tissue samples using Trizol. Extracted RNAs were resuspended in $5 \mu \mathrm{l}$ $\mathrm{ddH}_{2} \mathrm{O}$ and diluted ten times. RNA concentrations were determined using a NanoDrop ND-1000 spectrophotometer (Labtech, Palaiseau, France) and RNA integrity was assessed with an Agilent 2100 Bioanalyzer (Agilent, Massy, France). The mean RNA integrity number (RIN) was $7.94 \pm 0.6$ with a coefficient of variation $(\mathrm{CV})$ of $7.5 \%$. Samples with a RIN $<7.5$ were excluded from further analysis.

One microgram of total RNA was reverse transcribed using Superscript III and oligo(dT) primers (Invitrogen) according to the manufacturer's instructions. Real-time quantitative PCR was carried out using the SYBR green master mix (Applied Biosystems, Foster City, CA, USA) in a Mx 3005P thermocycler (Agilent Technologies). PCR conditions were $95^{\circ} \mathrm{C}$ for $10 \mathrm{~min}$, followed by 40 cycles of $95^{\circ} \mathrm{C}$ for $15 \mathrm{~s}$ and $60^{\circ} \mathrm{C}$ for $1 \mathrm{~min}$. At the end of the amplification reaction, melting curve analysis was performed to confirm the specificity as well as the integrity of the PCR product by the presence of a single peak. Optimal primer concentrations were determined for each target gene (see the Electronic Supplementary Table 1). Absence of crosscontamination and primer dimers was checked on genomic DNA and water. Four reference genes were tested for variable expression across the samples (Vandesompele et al. 2002). The most stably expressed genes were $\beta$-actin and $\beta 2$-microglobulin, and the geometric means of these two genes were chosen for normalization. Standard curves were generated from assays made with serial dilutions of reference cDNA to calculate PCR efficiencies $(100 \pm 15 \%$, with $\left.r^{2} \geq 0.997\right) . C_{\mathrm{t}}$ samples were transformed into quantity values using the formula $(1+\text { Efficiency })^{\mathrm{C}_{\mathrm{t}}}$. Only means of triplicate with a $\mathrm{CV}$ of $<10 \%$ were analyzed. Interplate variation was determined to be below $7 \%$.

Analysis of the expression of promoter-specific $I G F 2$ transcripts was performed by semi-quantitative RT-PCR (Claire Louet \& Hélène Jammes personal communication). Primers for the P1 were situated in exon 1 and exon 3 to yield a product of $352 \mathrm{bp}$. Primers for the P3 isoform were placed in exon 5 and on the exon junction $7 / 8$ to yield a $113 \mathrm{bp}$ amplification product, and a $439 \mathrm{bp}$ amplification product was obtained for the analysis of P4-specific transcripts using primers in exon 6 and 9.

\section{Analysis of LOI}

LOI was assessed by genotyping tumor DNA samples to identify informative samples for the ApaI polymorphism in exon 9 of $I G F 2$ (rs680). The ratio of the expressed alleles was quantitatively analyzed in the corresponding cDNA samples by pyrosequencing. Sequences of primers and modifications to the universal amplification protocol (see above) are given in the Electronic Supplementary Table 1. Genotyping and quantitative determination of allele ratios were carried out on the PSQ 96MD system with the PyroGold SNP Reagent Kit (Pyrosequencing), and results were analyzed using the PyroMark software (V1.0, Pyrosequencing AB).

\section{Immunohistochemistry}

We carried out immunohistochemical studies on paraffin sections using a tissue microarray (TMA) containing 28 insulinomas, 50 non-functioning PETs, and 10 peritumoral pancreatic specimens. TMAs were produced using a microarrayer (Beecher Instrument, Woodland, CA, USA). For each tumor, three cores of $0.8 \mathrm{~mm}$ in diameter were arrayed. For optimization of the assay and to avoid any confusion through the potential absence of islets of Langerhans on the small TMA sections, five normal pancreatic parenchymas were analyzed on standard paraffin sections. Sections were incubated with an antibody against IGF2 (clone W2H1, dilution: 1/50; Millipore SAS, Molsheim, France).

\section{Statistical analysis}

Differences in the distribution of methylation between two groups were assessed by the non-parametric Mann-Whitney or Kruskal-Wallis tests with $P<0.05$ considered as significant. 


\section{Results}

To investigate whether the hypermethylation of the IGF2 DMR2 that we recently reported in a mouse model of insulinomas (Fontaniere et al. 2006) is also present in human endocrine tumors, we analyzed the DNA methylation patterns of $40 \mathrm{CpGs}$ localized in the DMR0 (3 CpGs) and DMR2 (26 CpGs) of IGF2 and at the third CTCF-binding site (11 CpGs) of the H19 DMR (Fig. 1A) by pyrosequencing in a cohort of 62 patients with pancreatic and digestive tract endocrine tumors.

\section{Tissue-specific differences of IGF2 methylation}

The methylation patterns in the normal adjacent pancreatic tissues $(n=19)$ of the three different types of PETs (insulinomas, gastrinomas, and nonfunctioning PETs) showed on average the expected allele-specific methylation levels of $40-50 \%$ for the $I G F 2$ DMR0 and the two regions analyzed in the DMR2 (Fig. 1B). However, the methylation degree of individual $\mathrm{CpG}$ positions showed strong but very reproducible variations (20-70\%). No differences were detected between the normal adjacent tissues of the three tumor types. In contrast, the normal adjacent tissue of the SIETs $(n=11)$ displayed a significant higher methylation $(\sim 10 \%)$ compared with the pancreatic tissues for both regions of the IGF2 DMR2 analyzed (Fig. 1B; Table 2).

\section{Changes of IGF2 methylation in tumors}

Tumors were globally hypomethylated compared with their peritumoral tissues with the exception of the insulinomas (Fig. 1C and D, Table 2; Supplementary Table 2, which can be viewed online at http://erc. endocrinology-journals.org/supplemental/). Gastrinomas and non-functioning PETs showed a significant hypomethylation for the DMR0 (gastrinomas: $27.3 \%$, compared with the adjacent tissue $44.8 \%, P=0.0208$; non-functioning PETs 23.6 vs $47.7 \%, P=0.0005$; Fig. 2A). A certain degree of hypomethylation was also found in the DMR2, but this did not reach statistical significance (gastrinomas: DMR2a 34.6 vs 40.1\%, DMR2b 32.2 vs 37.2\%; non-functioning PETs: DMR2a 33.1 vs $40.6 \%$, DMR2b 32.5 vs $39.6 \%$ ). The hypomethylation was more pronounced in the SIETs (Fig. 2B). Statistically significant hypomethylation was observed for all DMRs in these tumors compared with the peritumoral tissues (DMR0 25.9 vs 53.6\%, $P=0.0003$; DMR2a 35.4 vs $52.5 \%, P<0.0001$; DMR2b 29.2 vs $47.8 \%, P=0.0008)$. The methylation level found in the SIETs was similar to those observed in the gastrinomas and non-functioning PETs.
In contrast to these three tumor types that exhibited different degrees of hypomethylation in the two IGF2 DMRs, the insulinomas were characterized by a hypermethylation compared with the normal adjacent tissue (Figs 2C and 3A; see the Electronic Supplementary Table 2). A trend towards higher methylation levels was observed in the DMR0 for the insulinomas (59.3 vs $47.4 \%)$. The hypermethylation was statistically significant for the two regions of the DMR2 (DMR2a 52.5 vs $41.7 \%, P=0.0188$; DMR2b 56.3 vs $39.9 \%, P=0.0018)$.

Thus, high levels of methylation in the IGF2 DMR2 and to a lesser extent DMR0 are a specific molecular alteration of insulinomas, which is not observed in any of the other tumor types analyzed.

\section{Methylation of the H19 DMR}

As the DMR upstream of $H 19$ is the ICR for the entire IGF2-H19 locus, we investigated whether methylation changes were also present in the DMR. The methylation patterns at the third CTCF-binding site were identical in the different peritumoral tissues, showing the expected allele-specific methylation levels $(46.4 \%$, Figs $1 \mathrm{~B}$ and 3B; see the Electronic Supplementary Table 2). The tissue-specific differences observed in the IGF2 DMRs were thus not present in the H19 DMR. Peritumoral samples from all tissues showed a reproducible methylation profile with increased methylation levels for $\mathrm{CpG}$ positions 2, 3, and 8, whereas the $\mathrm{CpG}$ positions underlying directly the CTCF-binding site (CpG positions 4-7) showed a consistent allele-specific methylation (44.6\%). Similarly, the methylation patterns were the same for all tumors independent of their origin $(42.2 \%)$. The same increase in methylation as in the peritumoral tissues was observed at the $\mathrm{CpG}$ position 8 , but not at the $\mathrm{CpG}$ positions 2 and 3. No significant changes were found in the average methylation level between normal and tumors for the different types of PETs. However, SIETs exhibited a significant difference between peritumoral $(46.3 \%)$ and tumoral tissues $(41.9 \%$; $P=0.0134)$. When analyzing the difference of methylation at individual $\mathrm{CpG}$ positions, $\mathrm{CpG}$ position 3 was significantly differentially methylated between peritumoral and tumoral samples in gastrinomas $(P=0.0088)$, non-functioning PETs $(P=0.0169)$, SIETs $(P=0.0095)$, and border line significant for insulinomas $(P=0.0522$; Fig. 2). However, changes were restricted to a single $\mathrm{CpG}$ position and it is unclear whether this changes leads to any functional consequences, especially as the 
Table 2 Statistical analysis of the methylation differences

\begin{tabular}{|c|c|c|c|c|}
\hline & IGF2 DMRO & IGF2 DMR2a & IGF2 DMR2b & H19 CTCF3 \\
\hline Insulinomas versus peritumoral tissues & NS & 0.0188 & 0.0018 & NS \\
\hline Gastrinomas versus peritumoral tissues & 0.0208 & NS & NS & NS \\
\hline Non-funct. PETs versus peritumoral tissues & 0.0005 & NS & NS & NS \\
\hline SIETs versus peritumoral tissues & 0.0003 & $<0.0001$ & 0.0008 & 0.0134 \\
\hline Insulinomas $\mathrm{P}$ versus gastrinomas $\mathrm{P}$ & NS & NS & NS & NS \\
\hline Insulinomas $\mathrm{T}$ versus gastrinomas $\mathrm{T}$ & 0.0012 & 0.0002 & 0.0003 & NS \\
\hline Insulinomas $\mathrm{P}$ versus non-funct. PETs $\mathrm{P}$ & NS & NS & NS & NS \\
\hline Insulinomas T versus non-funct. PETs T & 0.0002 & $<0.0001$ & $<0.0001$ & NS \\
\hline Insulinomas $\mathrm{P}$ versus SIETs $\mathrm{P}$ & NS & 0.009 & 0.0373 & NS \\
\hline Insulinomas T versus SIETs T & 0.0005 & 0.0002 & $<0.0001$ & NS \\
\hline Gastrinomas $P$ versus non-funct. PETs $P$ & NS & NS & NS & NS \\
\hline Gastrinomas T versus non-funct. PETs T & NS & NS & NS & NS \\
\hline Gastrinomas $P$ versus SIETs $P$ & NS & 0.0013 & 0.0248 & NS \\
\hline Gastrinomas T versus SIETs T & NS & NS & NS & NS \\
\hline Non-funct. PETs $P$ versus SIETs $P$ & NS & 0.0043 & 0.0790 & NS \\
\hline Non-funct. PETs T versus SIETs T & NS & NS & NS & NS \\
\hline
\end{tabular}

NS, not significant, figures in bold are statistically significant; $P$, peritumoral tissues; $T$, tumor tissues; SIETs, small intestine endocrine tumors; Non-funct. PETs, non-functioning pancreatic endocrine tumors.

CpG sites underlying the CTCF-binding site display the expected methylation profile in all tumoral and normal samples.

\section{Expression of IGF2}

To evaluate potential transcriptional consequences of the methylation changes in the IGF2 DMRs, expression analysis of IGF2 was performed in a subset of the above-described tumors samples by quantitative RT-PCR. IGF2 was found to be expressed in normal pancreatic tissue. The expression level was significantly different between normal pancreas, insulinomas, and the three other tumor types (Fig. 4A; Kruskal-Wallis test; $P=0.0005$ ), and insulinomas were also different when compared with the three other tumor types (Mann-Whitney test; $P=0.0016$ ).
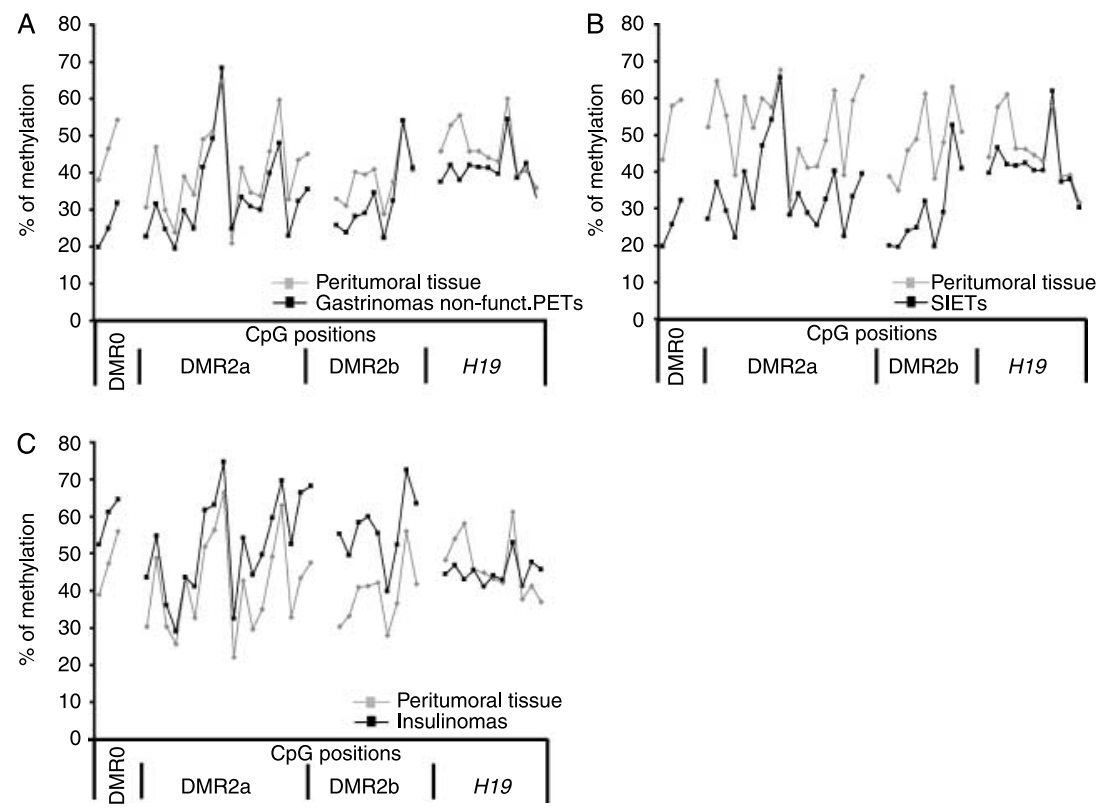

Figure 2 Detailed methylation patterns of the IGF2-H19 locus for tumors and their corresponding normal adjacent tissue. (A) Gastrinomas and non-functioning PETs (data from both groups were regrouped for better visualization), (B) small intestine endocrine tumors, and $(C)$ insulinomas. Data represent the mean of a tumor type or its peritumoral component for each CpG position. Details on the average DNA methylation degree for each region as well as the S.D. and range of methylation values are given in Supplementary Table 2, which can be viewed online at http://erc.endocrinology-journals.org/supplemental/. 

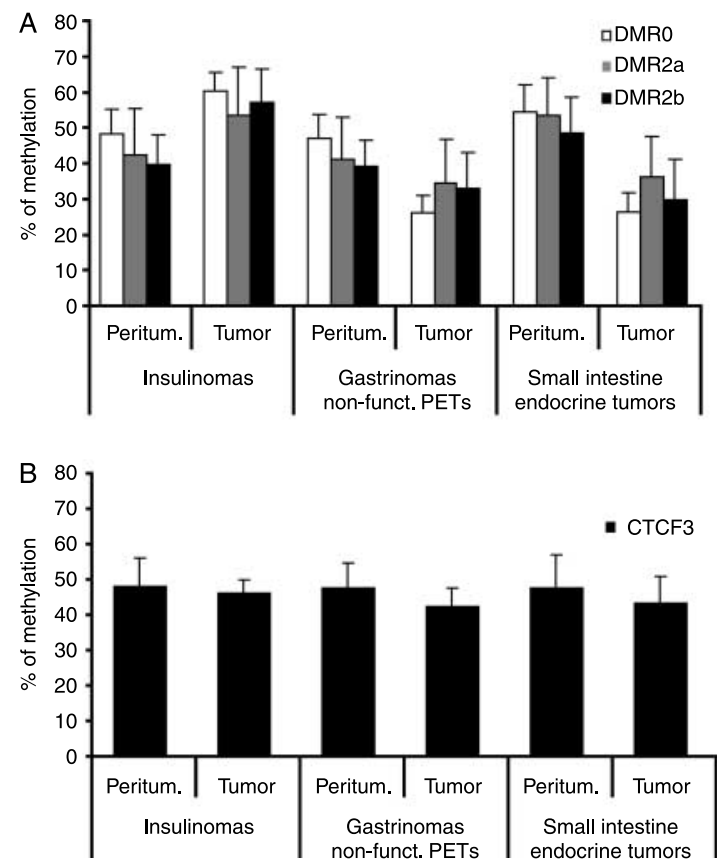

Figure 3 Mean methylation level (+S.D.) for IGF2 (A) and H19 (B) for each type of tumors and for each region analyzed (gastrinomas and non-functioning PETs are regrouped). The percentage of methylation corresponds to the average methylation level of the specific region. Details on the average DNA methylation degree for each region as well as the s.D. and range of methylation values are given in Supplementary Table 2, which can be viewed online at http://erc.endocrinology-journals. org/supplemental/.

An increased expression level was specifically found in insulinomas, while no difference in the level of expression was found between the other three tumor types (gastrinomas, non-functioning PETs and SIETs). The latter three were found to be less expressed in the tumoral samples compared with normal pancreatic tissue.

\section{LOI of IGF2}

As $I G F 2$ is an imprinted gene, we studied the possible LOI that might explain the increased expression of $I G F 2$. Five insulinomas were heterozygous for the ApaI polymorphism in exon 9 of IGF2 and had biallelic expression of IGF2. 30-50\% of all transcripts could be attributed to the reactivation of the silent allele by the quantification of alleles in the cDNA. All these insulinomas were of uncertain behavior according to the WHO classification. Unfortunately, all other tumors studied (four gastrinomas, three nonfunctioning PETs, and three SIETs) were all homozygous for this SNP. These did, therefore, not permit to investigate whether hypomethylation is associated with a LOI.

\section{Promoter-specific expression analysis}

As IGF2 expression is regulated by different promoters, we searched for the promoter responsible for the biallelic overexpression. We therefore analyzed the contribution of the promoters P1 (transcript fusing exons 1, 2, 3, (4), 7, 8, and 9), P3 (exons 5, 7, 8, and 9), and P4 (exons 6, 7, 8, and 9) to IGF2 expression using semi-quantitative RT-PCR with primers specific for the different isoforms. We did not observe consistent amplification using the P3 primers. However, promoters P1 and $\mathrm{P} 4$ gave clear amplification products of the expected size (Fig. 4B). In a commercial liver sample, we found as expected IGF2 mRNA expressed from the $\mathrm{P} 1$ promoter, while in normal pancreas IGF2 was expressed only from the $\mathrm{P} 4$ and not the P1 promoter. Gastrinomas and non-functioning PETs did not display expression from either promoter or low expression from the $\mathrm{P} 4$ as expected from the total IGF2 mRNA measurements. One insulinoma was strongly and exclusively expressed from the P1 promoter, two insulinomas had expression from both promoters with the $\mathrm{P} 4$ transcript being largely dominant, and two insulinomas were expressed only from the P4 promoter. Total mRNA levels correlated well with the band intensity from the $\mathrm{P} 4$ promoter. No correlations between promoter usage and any molecular characteristics were found.

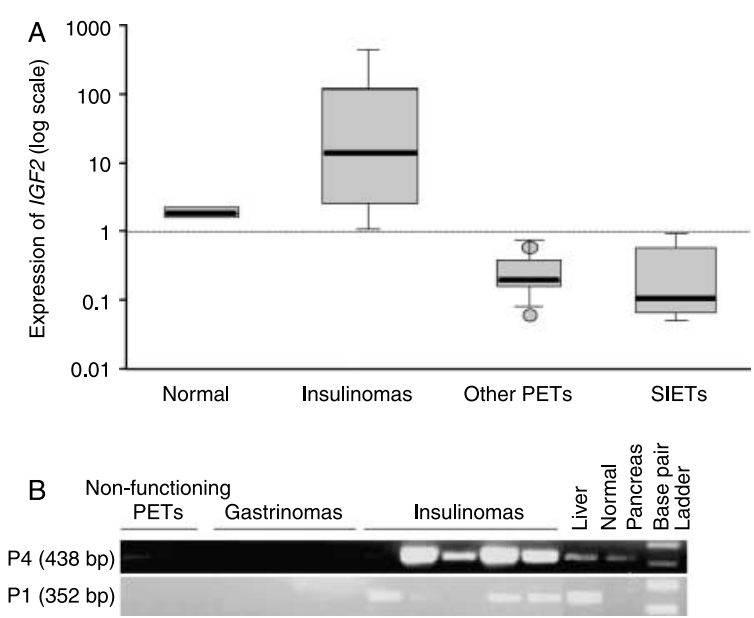

Figure 4 (A) Expression data are represented as a box plot with logarithmic scale. Data are normalized in function of geometric mean of the expression of two references genes ( $\beta$-actin and $\beta 2$-microglobulin). (B) Semi-quantitative analysis of promoterspecific IGF2 transcripts. P1 and/or P4 transcripts are highly expressed in insulinomas, but very weakly in non-functioning PETs and gastrinomas. 


\section{Immunohistochemistry for IGF2}

The antibody against IGF2 was tested in normal pancreatic tissue and stained weakly the normal islets of Langerhans (Fig. 5A). In contrast, high or moderated expression of IGF2 was observed in $50 \%$ of insulinomas (14 out of 28 cases tested, Fig. 5B). In non-functioning PETs, IGF2 expression was only detectable in $16 \%$ of the tumor specimens ( 8 out of 50 samples tested, Fig. 5C). Good correlation between the immunohistochemistry (IHC) and RT-qPCR data was found. The insulinomas with the highest protein expression in the tumors also had the highest level of RNA expression. However, detectable protein expression decreased rapidly for the weaker RNA expression values. No differences were observed for the staining of the peritumoral tissues compared with normal pancreatic tissues, indicating that the overexpression of IGF2 in the insulinomas is a tumorspecific event.

\section{Methylation is correlated with tumor prognostic}

We then investigated a possible correlation between the methylation patterns and the classification and staging system of PETs according to the WHO (benign, uncertain behavior, and well-differentiated endocrine carcinomas). A significant decrease in methylation level for the different DMRs of $I G F 2$ was correlating with the WHO classification, with the benign group displaying the highest degree of methylation (Fig. 6, Kruskal-Wallis test; $P=0.0068$ for DMR0; $P=0.0013$ for DMR2a; and $P=0.0015$ for DMR2b). In contrast, the methylation was stable for all three categories for the H19 DMR. However, when analyzing specifically insulinomas that were classified as either benign or of uncertain behavior according to the WHO classification, a small but not significant loss of methylation was observed for IGF2 DMR0 in the more advanced tumors, while the two analyzed regions of the DMR2 displayed the same hypermethylation in both groups of the tumors (see Supplementary Fig. 1, which can be viewed online at http://erc.endocrinologyjournals.org/supplemental/). Hypermethylation of the DMR2 is thus a stage-independent molecular alteration in insulinomas. To rule out that the stage-dependent loss of methylation observed in the other tumors is not caused by global changes at the methylation level in the tumors, we also studied the methylation of repetitive element $(A l u)$ by pyrosequencing as previously published (Yang et al. 2004). All PET samples were highly methylated at the Alu elements for peritumoral and tumoral samples without any significant differences in function of the origin or the stage of the tumor. Only the SIET samples to which the WHO classification does not apply showed a trend towards hypomethylation in the tumor samples compared with the peritumoral tissues (Mann-Whitney; $P=0.088$ ).

\section{Other molecular and clinical correlates}

We analyzed the methylation patterns for a potential correlation with the presence/absence of metastases. To avoid any confounding effect of the tumor-typespecific DNA methylation patterns as well as the tumor stage on the analysis, we investigated the potential influence only in gastrinomas and nonfunctioning PETs. No association between methylation patterns and the metastatic status of patients was found for any of the DMRs. As gastrinomas originated from either the pancreas itself or the duodenal wall, we analyzed whether any correlation between the origin of the tumor and the DNA methylation patterns could be observed. Methylation patterns from the two subtypes of gastrinomas were identical for all regions analyzed. Some patients with gastrinomas and nonfunctioning PETs developed tumors in association with a MEN1 syndrome. We therefore compared their methylation patterns to the sporadically developed
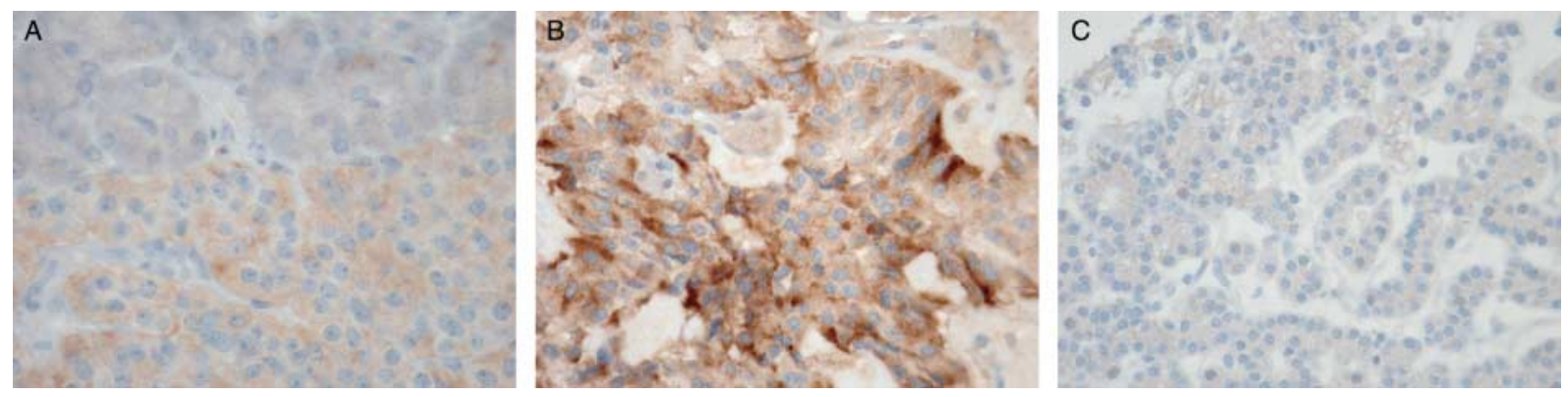

Figure 5 Immunohistochemistry on paraffin section using an IGF2 antibody. (A) Weak staining for IGF2 of the islets of Langerhans in a peritumoral tissue, (B) strong staining in an insulinoma, and (C) absence of staining in a non-functioning PET. Staining of normal pancreatic tissue was indistinguishable from the staining of peritumoral tissue. 
tumors of the same type. No significant differences were observed for the non-functioning PETs. The gastrinomas with a MEN1 background, however, showed significantly elevated methylation levels in the IGF2 DMR0 and DMR2b $(P=0.0339$ and $P=0.0317$ respectively), while the results were borderline significant for DMR2a $(P=0.0532)$. Whereas most tumors lose methylation compared with the peritumoral tissues, the tumors with a MEN1 syndrome display similar levels as the normal tissues. However, these results should be taken with care due to the low numbers of MEN1-associated tumors.

\section{Age-related methylation}

DNA methylation is known to change in response to environmental stimuli as well as with age. Notably, methylation of IGF2 has been demonstrated to increase with age in epithelial tissues such as the colon (Issa et al. 1996). Therefore, we investigated a potential correlation between age and methylation to rule out any confounding effects on our results due to an effect of age. Peritumoral samples displayed a weak correlation of the methylation level of the IGF2 DMR2a with age $\left(R^{2}=0.1452\right)$ (see Supplementary Fig. 2, which can be viewed
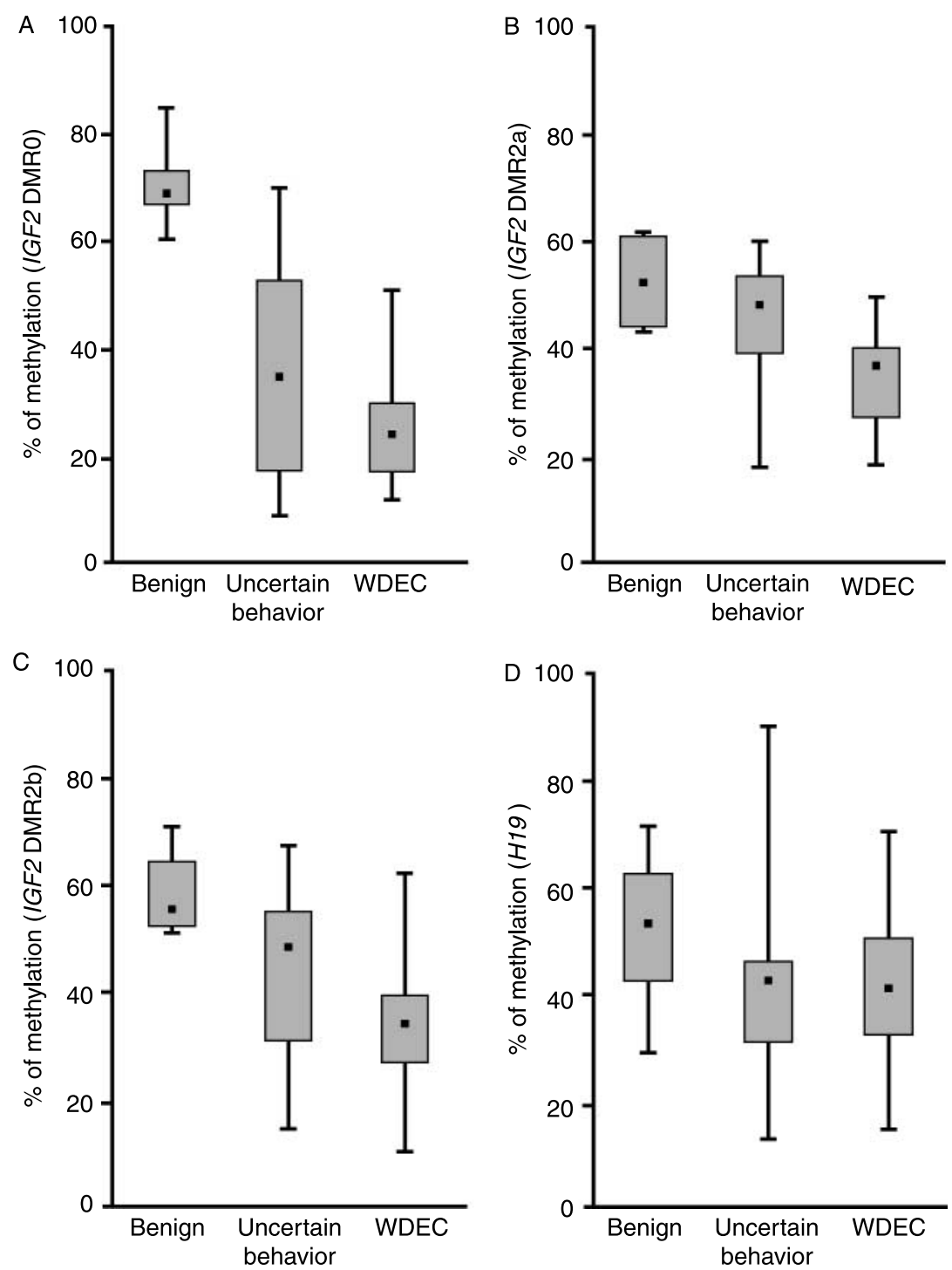

Figure 6 Methylation levels of the IGF2-H19 locus (A) IGF2 DMR0, (B) DMR2a, (C) DMR2b, and (D) H19 for all pancreatic endocrine tumors classified according to the WHO (benign, uncertain behavior, and well-differentiated endocrine carcinoma (WDEC)). 
online at http://erc.endocrinology-journals.org/supplemental/). However, no correlation between age and the observed methylation levels was detected for the pancreatic or SIETs. This clearly demonstrates that tumor-specific molecular events rather than aging determine the methylation levels in the different regions of $I G F 2$.

\section{Discussion}

In the present study, we analyzed the DNA methylation patterns of the IGF2-H19 locus in endocrine tumors of the pancreas and the digestive tract by pyrosequencing. The methylation profile of IGF2 displayed tissuespecific differences with the adjacent tissue of the SIETs being more methylated compared with the pancreatic tissue, which was identical for all three tumor types analyzed.

Insulinomas displayed a very particular DNA methylation profile. The increased methylation levels in the IGF2 DMR2 were found to specifically distinguish this type of tumor from the other PETs, which are characterized by a more or less pronounced loss of methylation in this region. The other three types of tumors analyzed (gastrinomas, non-functioning PETs, and SIETs) showed a significant hypomethylation of the IGF2 DMR0, which was not found in the insulinomas. Insulinomas of both benign and uncertain behavior displayed this constant hypermethylation of the DMR2 and to a lesser extent DMR0, clearly showing that this alteration is a hallmark of their tumorigenesis. Insulinomas were also the only group of analyzed tumors that consistently overexpressed $I G F 2$ at the RNA and protein, while all other tumor types had lower expression levels than normal pancreatic tissue. The overexpression of IGF2 was restricted to the tumoral component of the tissue, as peritumoral tissue exhibited the same immunohistochemistry staining as normal pancreatic tissue. The overexpression is caused by a LOI and reactivation of the normally silent maternal allele. P4-specific $I G F 2$ transcripts and to a lesser extent P1 transcripts accounted for the observed increase in $I G F 2$ transcription. The presence of a heterozygous polymorphism, which is located in exon 9 of IGF2 and thus close to the analyzed DMR in the cDNA, also precludes the loss of the maternal and duplication of the paternal allele as a possible alternative mechanism for the overexpression of $I G F 2$. This has, for example, been reported in adrenocortical tumors that are also characterized by IGF2 overexpression (Gicquel et al. 1997).

Gastrinomas, non-functioning PETs, and SIETs displayed a methylation profile, which is comparable with the one observed in many types of sporadic tumors with a stable methylation of the H19 DMR, but significant hypomethylation of at least the IGF2 DMR0 (Murrell et al. 2008). The hypomethylation of the DMR0 itself has been proposed as a DNA methylationbased biomarker for risk assessment of cancer development (Cui et al. 2003, Kaneda \& Feinberg 2005, Ito et al. 2008). Hypomethylation of the DMR0 is often - but not necessarily - associated with LOI (Ito et al. 2008). Many tumors retain a mono-allelic expression despite a significant hypomethylation. However, all cancers with LOI also displayed hypomethylation of the DMR0 (Ito et al. 2008). Unfortunately, none of the tumor samples other than the insulinomas were informative for the assessment of LOI. Our data, in a different tumor context of course, provide further evidence that loss of imprinting and DNA hypomethylation of the DMR0 changes might be two independent events as we observe LOI in the presence of hypermethylation of the DMR0. The DMR0 has been postulated to have promoter activity (Monk et al. 2006). Thus, increased methylation could lead to decreased promoter activity. However, we observed no expression of $I G F 2$ in the hypomethylated tumors and increased expression despite increased methylation in the insulinomas, which might indicate that the changes in the DMR0 methylation might be a surrogate for other molecular changes but without direct functional consequences.

DNA methylation changes in the DMR2 have, to our knowledge, not yet been assessed in human tumors, but preliminary data from our laboratory show a significant hypomethylation of this region in several types of human cancers including breast, liver, and colorectal tumors (J Tost unpublished observations). However, this hypomethylation is not consistently associated with changes at the transcriptional level.

The observed molecular changes of insulinomas at the DNA methylation and mRNA expression level are similar to those we previously described in a mouse model that develops insulinomas due to a pancreatic $\beta$-cell-specific knockout of Menl (Fontaniere et al. 2006). However, the genetic background of the mice did not allow the investigation of a potential LOI as observed in this study. As in the mouse model, the most significant changes of the DNA methylation patterns were located in the IGF2 DMR2. In the mouse model, the changes were restricted to the $\mathrm{CpG}$ positions that constitute the core region of the DMR2, where DNA methylation leads to increased expression through an augmentation of transcription initiation (Murrell et al. 2001). Whether the human DMR2 regulates $I G F 2$ expression through a similar mechanism remains unclear. The organization of the IGF2-H19 locus is similar between the two species, 
but there are some species-specific differences in elements participating in the regulation and establishment of a higher order chromatin structure at this imprinted locus. A DMR corresponding to the mouse DMR1 has not been identified in human. In mouse, the unmethylated maternal allele interacts with H19 DMR and places thereby $\operatorname{Ig} 2$ in a silent chromatin loop favoring $H 19$ expression (Murrell et al. 2004). The absence of this DMR in human requires therefore other so far unknown mechanisms to establish the long-range chromatin interactions. A second difference is found for the DMR0. This region is only differentially methylated in the mouse placenta, where methylation occurs on the maternal allele, while this region is methylated specifically on the paternal allele in human tissues (Murrell et al. 2008). Taking these differences into account, the conserved hypermethylation of the murine and human DMR2 indicates that the upregulation of IGF2 expression might be a major factor in the tumorigenesis of insulinomas.

The transcription of IGF2 is controlled by five promoters (Fig. 1) with P2-P4 being genomically imprinted and mainly expressed in fetal tissues ( $\mathrm{Li}$ et al. 1996). The $\mathrm{P} 1$ promoter regulates biallelic expression of IGF2 in adult liver and has been shown to be reactivated in some tumors (Issa et al. 1996). Promoter-specific expression analysis confirmed a partial expression from P1 specifically in some of the insulinomas. However, most of the total RNA could be attributed to transcripts from the $\mathrm{P} 4$ promoter, which were significantly increased in insulinomas compared with normal pancreatic tissue or other PETs. Upregulation of IGF2 expression through activation of fetal promoters has previously been reported in various cancers including hepatocellular carcinoma (Tang et al. 2006). This increased expression has so far not been correlated with DNA methylation changes in the DMR2, but only at the respective promoter sites. Hepatocellular carcinoma is characterized by a loss of methylation in the IGF2 DMR2 (J Tost unpublished observations), which correlates potentially with the reported hypomethylation of the activated promoters in carcinogenesis (Tang et al. 2006). Insulinomas, however, activate $\mathrm{P} 4$ expression through a different mechanism. Here, we provide evidence that hypermethylation of the DMR2 leads to - or correlates with - an increased expression of $I G F 2$ through biallelic activation of the $\mathrm{P} 4$ promoter. Thus, in analogy to the mouse model for $I g f 2$ regulation, hypomethylation of the promoter could directly increase transcription of the $\mathrm{P} 4$ promoter, while hypermethylation of the DMR2 might increase transcription initiation at the respective promoter.
Although patients with insulinomas did not display a MEN1 phenotype, we cannot exclude that some of them harbor inactivating point mutations in MEN1. However, taking the results presented in this study into account, it seems to be more likely that the observed Igf2 hypermethylation in the $M e n 1^{-/-}$models is part of the tumorigenesis of insulinomas rather than being directly induced by the Menl inactivation.

$I G F 2$ has been found to be upregulated through different mechanisms in many tumor types. As the activation of a strong oncogene such as k-ras or myc is not a common event in PETs (Oberg \& Eriksson 2005), it might well be that the autocrine growth-promoting properties of genes such as the IGFs enable and stimulate cell proliferation and tumorigenesis. However, insulinomas do apparently have a balancing mechanism compensating the upregulation, as this does not have deleterious consequences for the patient. While in the SV40 large T-antigen-induced insulinoma mouse model (Christofori et al. 1994, 1995) as well as in multiple tumor types such as gastrointestinal stromal tumors (Braconi et al. 2008), IGF2 overexpression contributes to tumor growth, increased mitotic indices, higher risk of relapse, and worse prognosis; insulinomas are benign tumors with a good prognosis.

Multiple studies have assessed molecular changes and prognosis of PETs (reviewed in Halfdanarson et al. (2008)). However, results are often confounded and skewed as different types of PETs have not been separately analyzed. For example, insulinomas and gastrinomas have often been categorized together as functioning PETs. However, our study clearly shows that they differ in terms of their molecular signature and probably in their mechanism of tumorigenesis. They should therefore be treated as different entities.

The understanding of the pathobiology of endocrine tumors as well as their clinical management is currently complicated by the difficulty to accurately assess tumor prognosis and disease outcome. The WHO classification provides some guidelines for the classification of PETs depending on molecular parameters such as tumor size, proliferative and hormone activity, angioinvasion, and metastases. Understanding the key molecular changes in the different types of PETs is thus a crucial step towards a better tumor classification, an improved diagnosis and prognosis, and a tumor-specific rationalized therapy. Here, we have established a tight correlation between the WHO classification and the methylation levels of different DMRs of $I G F 2$. DNA methylation decreases gradually with advanced tumor stage with the exception of the above-discussed methylation patterns of the DMR2 in insulinomas. Most tumors display a 
global hypomethylation of the genome, which has been suggested to initiate and propagate oncogenesis by inducing chromosome instabilities and transcriptional activation of oncogenes and pro-metastatic genes (Ehrlich 2002). However, the decrease in DNA methylation in $I G F 2$ is gene specific and not a surrogate for global DNA methylation changes, as demonstrated by the unaltered DNA methylation levels for Alu elements in the analyzed PETs. Our observations are in concordance with another recent study (Choi et al. 2007), which also showed that only endocrine tumors of the small intestine showed a trend towards hypomethylation.

The methylation patterns of IGF2 offer therefore a molecular marker for the better understanding of the heterogeneous evolutionary potential of PETs. Larger prospective studies requiring probably multicenter collaborations due to the rare occurrence of these tumors will be necessary to fully evaluate the value of this novel promising DNA methylation-based biomarker compared with the WHO classification.

\section{Declaration of interest}

The authors declare that there is no conflict of interest that could be perceived as prejudicing the impartiality of the research reported.

\section{Funding}

This work was supported by the EU framework 6 integrated project MolPAGE (LSHG-CT-2004-512066) and the French Ministry of Research.

\section{Acknowledgements}

We thank Hafida El abdalaoui for the bisulfite treatment of the samples, Anne Boland-Auge for the measure of DNA concentration, and Florence Busato for technical assistance. We are grateful to Claire Louet and Hélène Jammes (INRA, Jouy-en-Josas, France) for sharing the protocols for the promoter-specific amplification of the $I G F 2$ isoforms.

\section{References}

Bjornsson HT, Brown LJ, Fallin MD, Rongione MA, Bibikova M, Wickham E, Fan JB \& Feinberg AP 2007 Epigenetic specificity of loss of imprinting of the IGF2 gene in Wilms tumors. Journal of the National Cancer Institute 99 1270-1273.

Braconi C, Bracci R, Bearzi I, Bianchi F, Sabato S, Mandolesi A, Belvederesi L, Cascinu S, Valeri N \& Cellerino R 2008 Insulin-like growth factor (IGF) 1 and 2 help to predict disease outcome in GIST patients. Annals of Oncology 19 1293-1298.
Chan AO, Kim SG, Bedeir A, Issa JP, Hamilton SR \& Rashid A $2003 \mathrm{CpG}$ island methylation in carcinoid and pancreatic endocrine tumors. Oncogene 22 924-934.

Choi IS, Estecio MR, Nagano Y, Kim do H, White JA, Yao JC, Issa JP \& Rashid A 2007 Hypomethylation of LINE-1 and Alu in well-differentiated neuroendocrine tumors (pancreatic endocrine tumors and carcinoid tumors). Modern Pathology 20 802-810.

Christofori G, Naik P \& Hanahan D 1994 A second signal supplied by insulin-like growth factor II in oncogeneinduced tumorigenesis. Nature 369 414-418.

Christofori G, Naik P \& Hanahan D 1995 Deregulation of both imprinted and expressed alleles of the insulin-like growth factor 2 gene during beta-cell tumorigenesis. Nature Genetics 10 196-201.

Cui H, Cruz-Correa M, Giardiello FM, Hutcheon DF, Kafonek DR, Brandenburg S, Wu Y, He X, Powe NR \& Feinberg AP 2003 Loss of IGF2 imprinting: a potential marker of colorectal cancer risk. Science 299 1753-1755.

Eggermann T, Eggermann K \& Schonherr N 2008 Growth retardation versus overgrowth: Silver-Russell syndrome is genetically opposite to Beckwith-Wiedemann syndrome. Trends in Genetics 24 195-204.

Ehrlich M 2002 DNA methylation in cancer: too much, but also too little. Oncogene 21 5400-5413.

Fontaniere S, Tost J, Wierinckx A, Lachuer J, Lu J, Hussein N, Busato F, Gut I, Wang ZQ \& Zhang CX 2006 Gene expression profiling in insulinomas of Men 1 beta-cell mutant mice reveals early genetic and epigenetic events involved in pancreatic beta-cell tumorigenesis. Endocrine-Related Cancer 13 1223-1236.

Gicquel C, Raffin-Sanson ML, Gaston V, Bertagna X, Plouin PF, Schlumberger M, Louvel A, Luton JP \& Le Bouc Y 1997 Structural and functional abnormalities at $11 \mathrm{p} 15$ are associated with the malignant phenotype in sporadic adrenocortical tumors: study on a series of 82 tumors. Journal of Clinical Endocrinology and Metabolism 82 2559-2565.

Gicquel C, Rossignol S, Cabrol S, Houang M, Steunou V, Barbu V, Danton F, Thibaud N, Le Merrer M, Burglen L et al. 2005 Epimutation of the telomeric imprinting center region on chromosome 11p15 in Silver-Russell syndrome. Nature Genetics 37 1003-1007.

Grant CS 2005 Insulinoma. Best Practice \& Research. Clinical Gastroenterology 19 783-798.

Halfdanarson TR, Rubin J, Farnell MB, Grant CS \& Petersen GM 2008 Pancreatic endocrine neoplasms: epidemiology and prognosis of pancreatic endocrine tumors. Endocrine-Related Cancer 15 409-427.

House MG, Herman JG, Guo MZ, Hooker CM, Schulick RD, Cameron JL, Hruban RH, Maitra A \& Yeo CJ $2003 a$ Prognostic value of hMLH1 methylation and microsatellite instability in pancreatic endocrine neoplasms. Surgery 134 902-908.

House MG, Herman JG, Guo MZ, Hooker CM, Schulick RD, Lillemoe KD, Cameron JL, Hruban RH, Maitra A \& 
Yeo CJ 2003b Aberrant hypermethylation of tumor suppressor genes in pancreatic endocrine neoplasms. Annals of Surgery 238 423-431.

Issa JP, Vertino PM, Boehm CD, Newsham IF \& Baylin SB 1996 Switch from monoallelic to biallelic human IGF2 promoter methylation during aging and carcinogenesis. PNAS 93 11757-11762.

Ito Y, Koessler T, Ibrahim AE, Rai S, Vowler SL, Abu-Amero S, Silva AL, Maia AT, Huddleston JE, Uribe-Lewis S et al. 2008 Somatically acquired hypomethylation of IGF2 in breast and colorectal cancer. Human Molecular Genetics 17 2633-2643.

Jones PA \& Baylin SB 2007 The epigenomics of cancer. Cell 128 683-692.

Jones JI \& Clemmons DR 1995 Insulin-like growth factors and their binding proteins: biological actions. Endocrine Reviews 16 3-34.

Kaneda A \& Feinberg AP 2005 Loss of imprinting of IGF2: a common epigenetic modifier of intestinal tumor risk. Cancer Research 65 11236-11240.

Klöppel G, Perren A \& Heitz PU 2004 The gastroenteropancreatic neuroendocrine cell system and its tumors: the WHO classification. Annals of the New York Academy of Sciences 1014 13-27.

Lewis A \& Reik W 2006 How imprinting centres work. Cytogenetic and Genome Research 113 81-89.

Li X, Cui H, Sandstedt B, Nordlinder H, Larsson E \& Ekstrom TJ 1996 Expression levels of the insulin-like growth factor-II gene (IGF2) in the human liver: developmental relationships of the four promoters. Journal of Endocrinology 149 117-124.

Liu L, Broaddus RR, Yao JC, Xie S, White JA, Wu TT, Hamilton SR \& Rashid A 2005 Epigenetic alterations in neuroendocrine tumors: methylation of RAS-association domain family 1, isoform A and p16 genes are associated with metastasis. Modern Pathology 18 1632-1640.

Monk D, Sanches R, Arnaud P, Apostolidou S, Hills FA, Abu-Amero S, Murrell A, Friess H, Reik W, Stanier P et al. 2006 Imprinting of IGF2 P0 transcript and novel alternatively spliced INS-IGF2 isoforms show differences between mouse and human. Human Molecular Genetics 15 1259-1269.
Murrell A, Heeson S, Bowden L, Constancia M, Dean W, Kelsey G \& Reik W 2001 An intragenic methylated region in the imprinted Igf2 gene augments transcription. EMBO Reports 2 1101-1106.

Murrell A, Heeson S \& Reik W 2004 Interaction between differentially methylated regions partitions the imprinted genes Igf2 and H19 into parent-specific chromatin loops. Nature Genetics 36 889-893.

Murrell A, Ito Y, Verde G, Huddleston J, Woodfine K, Silengo MC, Spreafico F, Perotti D, De Crescenzo A, Sparago A et al. 2008 Distinct methylation changes at the IGF2-H19 locus in congenital growth disorders and cancer. PLOS ONE 3 e1849.

Oberg K \& Eriksson B 2005 Endocrine tumours of the pancreas. Best Practice and Research. Clinical Gastroenterology 19 753-781.

Reik W, Dean W \& Walter J 2001 Epigenetic reprogramming in mammalian development. Science 293 1089-1093.

Serrano J, Goebel SU, Peghini PL, Lubensky IA, Gibril F \& Jensen RT 2000 Alterations in the p16INK4a/CDKN2A tumor suppressor gene in gastrinomas. Journal of Clinical Endocrinology and Metabolism 85 4146-4156.

Tang SH, Yang DH, Huang W, Zhou HK, Lu XH \& Ye G 2006 Hypomethylated P4 promoter induces expression of the insulin-like growth factor-II gene in hepatocellular carcinoma in a Chinese population. Clinical Cancer Research 12 4171-4177.

Tost J \& Gut IG 2007 DNA methylation analysis by pyrosequencing. Nature Protocols 2 2265-2275.

Vandesompele J, De Preter K, Pattyn F, Poppe B, Van Roy N, De Paepe A \& Speleman F 2002 Accurate normalization of real-time quantitative RT-PCR data by geometric averaging of multiple internal control genes. Genome Biology 3 R34.

Yang AS, Estecio MR, Doshi K, Kondo Y, Tajara EH \& Issa JP 2004 A simple method for estimating global DNA methylation using bisulfite PCR of repetitive DNA elements. Nucleic Acids Research 32 e38.

Zikusoka MN, Kidd M, Eick G, Latich I \& Modlin IM 2005 The molecular genetics of gastroenteropancreatic neuroendocrine tumors. Cancer 104 2292-2309. 